\title{
Numerical Simulation Study Of Squeezed Branch Piles Based On Post-grouting Technology
}

\author{
LiXing Ma ${ }^{1, *}$, ZhuoPeng Shi ${ }^{1}$, Kai Han ${ }^{1}$, PengFei Chang ${ }^{1}$, XiaoDong He ${ }^{1}$. \\ ${ }^{1} 1$ Economic and Electrical Research Institute of Shanxi Electrical Power Company of SGCC,030021 Taiyuan City,Shanxi \\ Province, China
}

\begin{abstract}
The squeezed branch pile has strong resistance to pressure, pullout and horizontal force, and it also has excellent stability, which makes it widely used in the field of electrical engineering. Based on the ABAQUS, the paper simulates the vertical bearing situation of post-grouting squeezed branch piles, by setting up Set two comparisons of single pile and squeezed branch pile, the bearing capacity, load transfer mechanism and failure mode of post-grouting squeezed branch piles were comprehensively analyzed and compared. Studies have shown that the bearing capacity of the squeezed branch piles with post-grouting technology has increased by $68.1 \%$ compared with the traditional squeezed branch piles, and the bearing characteristics of the branches have changed greatly. In this process, the end-bearing function of the branch plate is fully exerted, and the high load-bearing capacity of the grouted strata soil is used to reduce the settlement.
\end{abstract}

\section{Introduction}

The squeezed branch pile is a new type of pile foundation that has developed rapidly in recent years, and it is a kind of bored pile [1-4]. After the traditional cast-in-place pile construction is completed, the professional squeezing and expanding equipment is used to locate at the design depth according to the design and construction requirements, and the squeezing and expanding arms are repeatedly squeezed to form a branch or bearing plate. The squeezed branch and plate piles make full use of the bearing characteristics of the end bearing of the branch and plate, and transmit the pile top load to the compressive soil layer at the bottom of the plate one by one, reducing the axial force of the pile body and greatly improving the bearing capacity of a single pile. It also has a great effect in controlling settlement, with good pull resistance and ability to resist horizontal loads.

In recent years, due to the excellent characteristics of the squeezed branch piles, more and more scholars and experts have begun to get involved in the study of the bearing performance of the squeezed branch piles, and the results have been applied to turned into good economic benefits [5-6]. Throughout the previous research results, the main focus is on the study of the force performance, failure behavior and load transfer law of the squeezed branch pile. There are also many researchers who have made breakthroughs in the design and optimization of the branch and plate [7]. This paper mainly studies the bearing and failure properties of post-grouting squeezed branch piles, that is, after the squeezed branch piles are formed into piles, the pre-buried post-grouting equipment is used to squeeze the bottom of the branch, and the grout will infiltrate the soil at the bottom of the branch.
Compaction and splitting, the grout makes the bottom soil of the pan gradually cement and solidify to form a new stratum environment, which changes the load-bearing mode of traditional squeezed branch piles and improves the load-bearing capacity.

\section{Numerical simulation of bearing capacity of post-grouting squeezed branch pile}

\subsection{Basic assumptions and establishment of the model}

In this paper, the classic Mohr-Counlomb model is used to simulate the mechanical properties of the soil to carry out the analysis and calculation of the ultimate bearing capacity (strength problem), so as to realize the accurate expression of accuracy and reliability.

The Mohr-Counlomb model criterion is widely used in general geotechnical conditions, and its shear strength formula expression is shown in formula 1;

$$
\tau_{n}=c-\sigma_{n} \tan \varphi
$$

The yield surface of the Mohr-Counlomb model is an irregular hexagonal cross-section pyramid surface as shown in Figure 1, and its projection on the $\pi$ plane is shown in Figure 2:

\footnotetext{
*LiXing Ma: 646769909@qq.com
} 


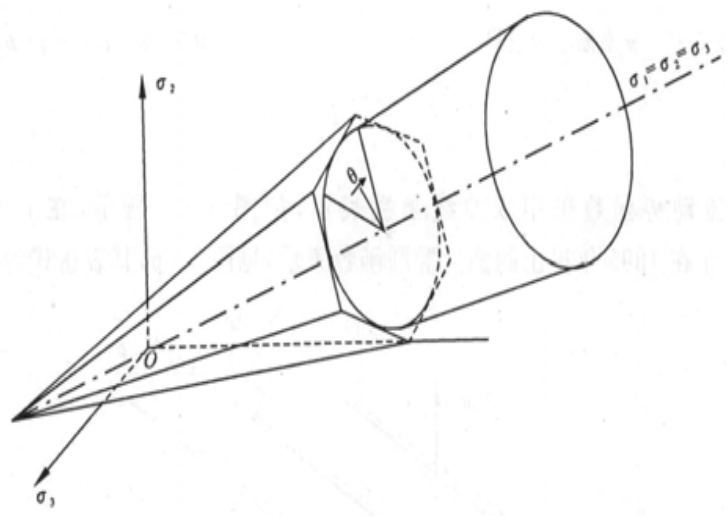

Figure 1. Mohr-Counlomb Model yield surface

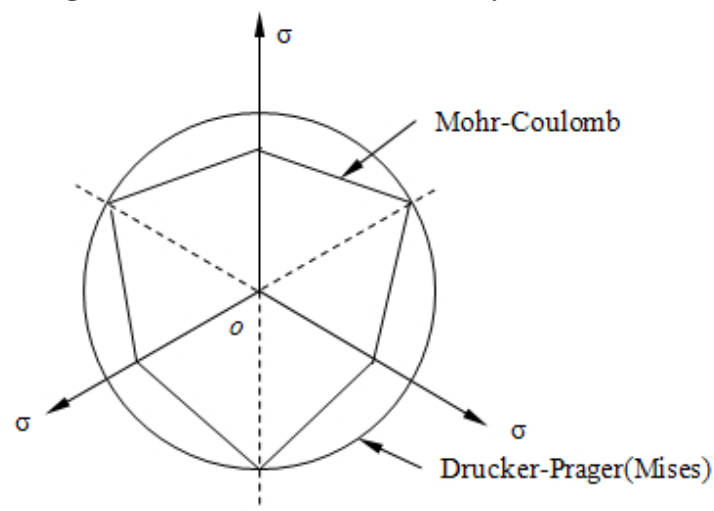

Figure 2. $\pi$ Plane projection

Since the slurry diffusion process is very complicated, we only consider the osmotic compaction of the sand layer. Based on the spherical diffusion law of grout in uniform and isotropic media, Maag deduced the formula of Newtonian grout permeation in sandy soil in 1938. So we built a partial sphere embedded in the bottom of the branch to simulate the Situation after grouting [8]. In the case of grouting behind the bottom, the grouting volume of the spherical body meets the grouting volume requirements of the Technical Specification for Building Pile Foundations.

In order to study the load transfer performance of postgrouting squeezed branch piles, this paper uses ABAQUS to carry out simulation analysis, establishes two comparison groups of traditional pile and ordinary squeezed branch pile, and then apply hierarchical uniform loads to the pile tops of the three models, observe the load transfer mode and failure behavior of the post-grouting squeezed branch pile under vertical compression.

\subsection{Finite element calculation results and load transfer mechanism analysis}

\subsubsection{Analysis of load-settlement relationship of single pile}

In this paper, according to the above steps, the vertical load on the top of the pile was applied to the three groups of models. The establishment work began to perform finite element calculation and analysis. There were no discontinuous iterations in the calculation process monitoring, and the convergence was good. In the postprocessing module, extract and output the load-settlement relationship curve of the top of a single pile. Similarly, extract the Q-s curve comparison analysis of the remaining two piles as shown in Figure 3.

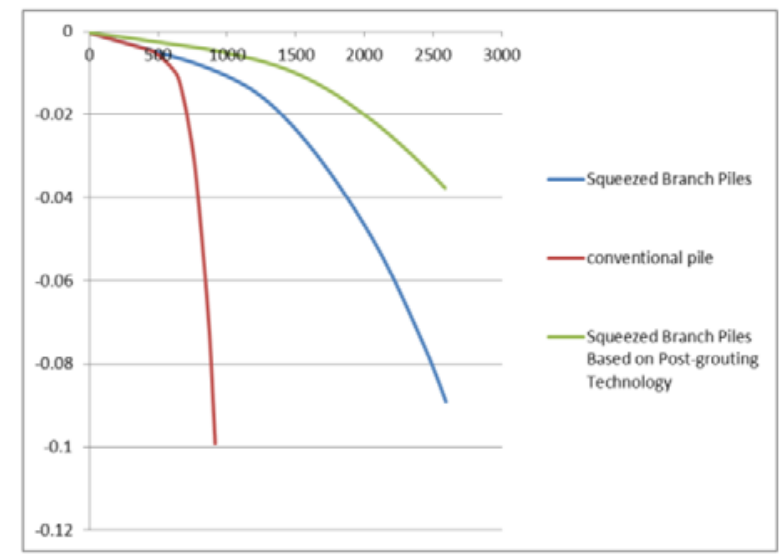

Figure 3. Q-s curve of three models

Table1. Model bearing capacity comparison

\begin{tabular}{|c|c|c|c|}
\hline & $\begin{array}{c}\text { traditional } \\
\text { pile }\end{array}$ & $\begin{array}{c}\text { squeezed } \\
\text { branch pile }\end{array}$ & $\begin{array}{c}\text { post-grouting } \\
\text { squeezed } \\
\text { branch pile }\end{array}$ \\
\hline $\begin{array}{c}\text { Ultimate } \\
\text { bearing } \\
\text { capacity } \\
(\mathrm{kN})\end{array}$ & 416.47 & 1251.6 & 2104.45 \\
\hline
\end{tabular}

It can be seen from the table that under the same stratum environment, the bearing capacity of the postgrouting squeezed branch pile is $2104.45 \mathrm{kN}$, which is $68.14 \%$ higher than the traditional squeezed branch pile, and the bearing capacity enhancement effect is very significant. From the perspective of the Qs curve trend of the three pile types, before $400 \mathrm{kN}$, the curves of the traditional pile and the squeezed branch pile basically overlap, showing a straight line trend, indicating that the pile body and the soil are in the elastic stage without yielding, the settlement value of post-grouting squeezed branch piles is significantly lower than that of the traditional squeezed branch pile. At about $500 \mathrm{kN}$, because the bottom of the pile is silty clay, the end resistance of the straight pile is very small and it is easy to pierce and fail. The curve shows the characteristic of steep decline. The Q-s curve of the post-grouting squeezed branch pile shows a linear change until $1000 \mathrm{kN}$, and the settlement is very regular and the value is very small. As the settlement continues to increase, the soil then enters the plastic deformation stage, and the squeezed branch piles and post-grouting squeezed branch piles have reached the ultimate bearing capacity and cannot continue to bear the load.

\subsubsection{Load transfer mechanism and failure analysis of post-grouting squeezed branch pile}

From Figure 2-5, it can be found that the general trend of the axial force transmission law of the post-grouting squeeze-expanded branch-plate pile is decreasing from the top of the pile to the bottom of the pile, and the axial force is drastically reduced at the position of the branch, but Under the first and second levels of load, the vertical stress value of the lower half of the pile body appears negative, indicating that the pile section has tensile stress, 
that is, the pile has negative friction under the first two levels of load.

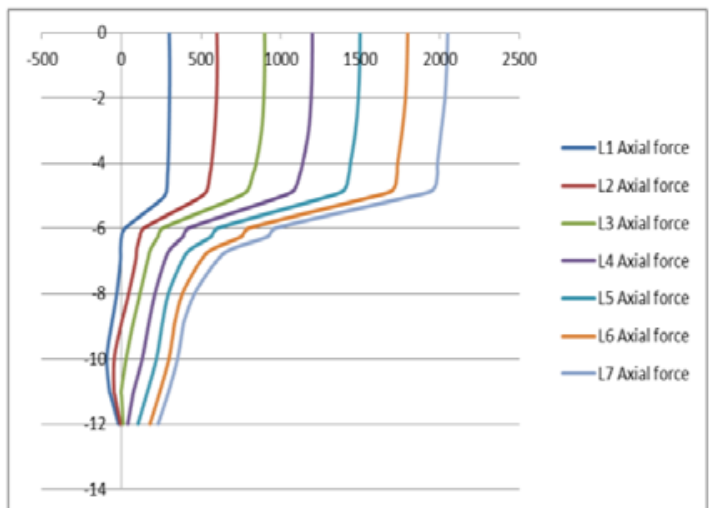

Figure 4. Axial force distribution diagram of post-grouting squeezed branch pile

Comparing the Q-s curve of post-grouting squeezed branch pile, it is found that the pile settlement is less than $2 \mathrm{~mm}$ under the first-level load. Because the area of the branch and plate increases after grouting, the pile top load at the initial stage of loading Almost all is borne by the support branch and the upper side resistance, and the reinforcement area of the support branch greatly limits the settlement value. The pile tip resistance and the lower side friction resistance have not yet been exerted. The lower half of the pile body overcomes the lower side friction under the weight of the concrete. Resistance, and produce a downward movement trend, causing the lower half of the pile to appear as tensile stress locally.

\subsubsection{Bearing characteristics of the reinforcement area of the support branch}

Comparing the Q-s curves of traditional squeezed branch piles and post-grouting squeezed branch piles, we find that before $500 \mathrm{kN}$, the settlement rate of post-grouting squeezed branch piles is significantly lower than that of traditional squeezed branch piles, which shows that the area of branch reinforcement has made a great contribution to the settlement of the pile foundation in the early stage. Next, analyze the bearing effect of the support branch reinforcement area, side friction resistance and bottom resistance, as shown in Figure 5 and Figure 6.

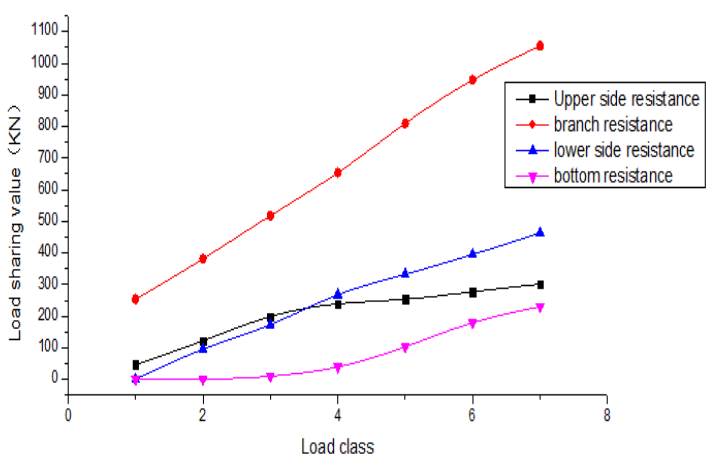

Figure 5. Post-grouting squeezed branch pile load sharing value

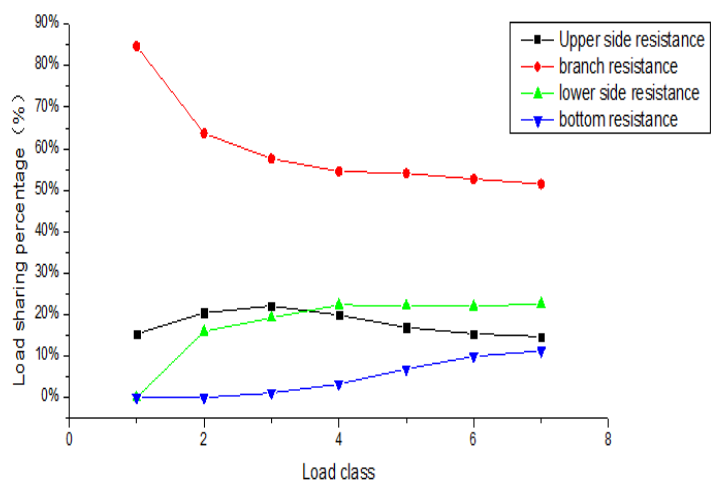

Figure 6. Post-grouting squeezed branch pile load sharing percentage

From the data extracted from the above figure, we can see that the branch reinforcement area has changed the load-bearing mode of the traditional squeezed branch piles, and the time and sequence of the load-bearing performance of each section has also changed.

Under the first-level load with a load of $300 \mathrm{kN}$ on the top of the pile, the support and branch reinforcement area took the lead in sharing the load, not only bearing $84.6 \%$ of the load value, but also controlling the settlement of the pile top within $2 \mathrm{~mm}$. When the load reaches the sixth level, the bearing capacity exerted by the reinforcement area of the support branch tends to be gentle and no longer increases, and the end resistance has been significantly improved. As the load of each section of the side resistance and the end resistance gradually increases, the strength of the reinforcement area of the support branch is gradually increased. The impact slowly weakened, the load sharing percentage dropped to $51.4 \%$, more than half of the total load, and the pile tip resistance only accounted for $11.23 \%$.

From the above analysis, compared with the traditional squeezed branch piles, the branch reinforcement area occupies a dominant position in the early stage of stress. It not only shares most of the load increment but also effectively reduces the relative displacement of the pile and soil. When the top load reaches $2100 \mathrm{kN}$, the end resistance of the branch and the pile end accounted for $62.7 \%$ of the total load.

\subsubsection{Bearing characteristics of pile side friction}

From the above figure, the performance of the upper side friction resistance of $0 \mathrm{~m}-5.1 \mathrm{~m}$ and the lower side friction resistance of $6 \mathrm{~m}-12 \mathrm{~m}$ is also slightly different from that of traditional squeezed branch piles. In the initial stage of loading, the load on the top of the pile is mainly borne by the branch reinforcement area and the upper side friction. Due to the small displacement of the pile, the lower side friction is not fully utilized. During the loading process from the second stage to the fourth stage, the lower side friction resistance increases steadily and the growth rate is basically the same as the upper side friction resistance, and both share the pile top load. When the fourth level of load is reached, the upper side friction resistance increases slowly and reaches the peak value, the load bearing percentage also decreases, and the lower side friction 
resistance begins to play, exceeding the upper side friction resistance and assuming $22 \%$ of the external load. This shows that The side-to-side grouting technology improves the side friction resistance of the pile to a certain extent, which is conducive to the development of the bearing capacity of the pile foundation.

\subsection{Analysis of soil vertical displacement}

The vertical displacement field of the soil layer of the post-grouting squeezed branch pile is shown in Figure 7. From the figure, we can see that the displacement field in the early stage of loading is evenly distributed and relatively stable, and most of the deformation occurs in Bottom and top of grouting reinforcement zone. From the perspective of the expansion trend, the position of the bottom surface and the top surface basically spread out around the existing displacement field, and the angle is gradually increasing. As the distance from the pile increases, the displacement value appears to be stratified and the value becomes smaller. The final vertical displacement field can be concluded that the displacement mainly occurs around the post-grouting reinforcement area, and the overall profile is similar to a "gourd" shape.

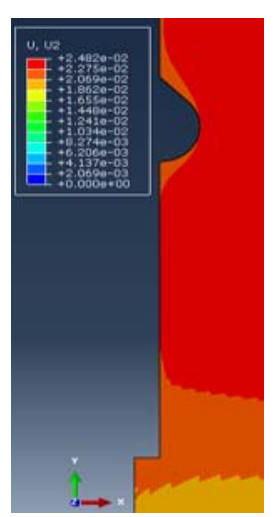

Load 300kN

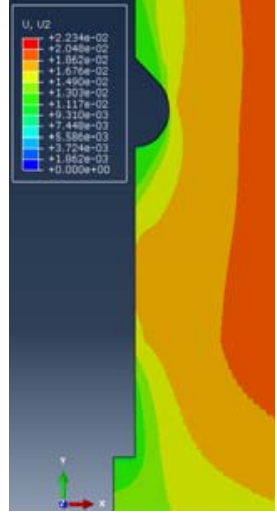

Load $1200 \mathrm{kN}$

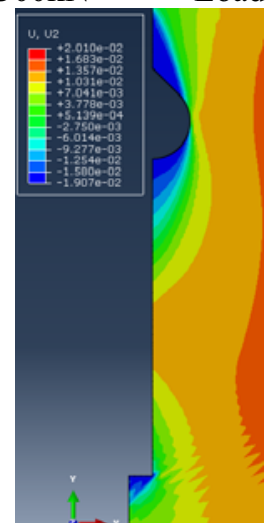

Load $2100 \mathrm{kN}$

Figure 7. Displacement field distribution of soil after postgrouting and expanding branch pile

\section{Conclusions}

(1) Under the same stratum environment, the bearing capacity of the post-grouting squeezed branch pile is $2104.45 \mathrm{kN}$, which is $68.14 \%$ higher than the traditional squeezed branch pile, and the bearing capacity enhancement effect is very significant. The Q-s curve of the post-grouting squeezed branch pile shows a linear change until $1000 \mathrm{kN}$, the settlement is very regular and the value is small, and the characteristics of slow change are more obvious.

(2) In terms of load transfer under vertical compression, the overall trend is to decrease from the top of the pile to the bottom of the pile. Axial forces are drastically reduced at the location of the support branch, and the support branch reinforcement area always dominates. From $86 \%$ under the first-level load to the final $51.4 \%$, which is more than half of the total load, the "Hold-up effect" performance is very outstanding and the settlement is effectively controlled.

(3) The post-grouting reinforcement area increases the contact area with the surrounding soil layer, and the reinforcement of the soil makes the Hold-up effect of the branch more obvious. The initial settlement value is controlled within $2 \mathrm{~mm}$, and finally as the settlement increases. The shear zone begins to extend along the weak soil layer around the support and branch reinforcement area, and the width increases and finally forms a stripshaped shear zone surrounding the support and branch reinforcement area. The displacement area of the reinforcement area and the bottom of the pile also began to expand slowly, and the overall outline resembled a "gourd" shape. Finally, the punching-shear zone soil at the outer edge of the reinforcement area was plastically deformed, and the soil at the end of the pile also began to lose strength, and the overall performance is shear failure.

\section{References}

1. Z.P. Jiang, W. Ming, Y.H.He,H.J.Kuang. Concrete and cement products. 10. 39-43. (2015)

2. D.Mohan,.V N S\&Jain GS.Murthy, Design and Construction of Multi-under Reamed piles, (1969)

3. D.Mohan, Bearing Capacity of Multi-under Reamed Piles, (1967)

4. Bruce,International Journal of Rock Mechanics \& Mining Sciences \& Geomechanics Abstracts,915,(1986)

5. X.D. Fu. Bridge construction. 10. 52-55. (1999)

6. J.L. Liu, J.C. Zhu. Building Science.02.13-18. (1996)

7. Simek.J, Verfel.J. Sedlecky.O, Holousova.T,Proceedings of the International Conference on soil Mechanics and Foundation Engineering, 1031-1034,(1989)

8. Fleming,W.G.K.The Improment of Pile Performance by Base Grouting. 88-93,(1993) 